OPEN ACCESS

Citation: L. Corbetta, L. M. Fabbri, D. Halpin, A. A. Cruz, S. Zanconato (2021) Stand on the Same Side Against Covid-19: Ics-Containing Products in Treatment Covid-19 Patients with Asthma and COPD. Substantia 4(1) Suppl. 1: 1233. DOI:

10.36253/Substantia-1234

Received: Feb 04, 2021

Revised: Mar 22, 2021

Just Accepted Online: Mar 22, 2021

Published: Mar 22, 2021

Copyright: () 2021 L. Corbetta, L. M. Fabbri, D. Halpin, A. A. Cruz, S. Zanconato. This is an open access, peer-reviewed article published by Firenze University Press (www.substantia.net) and distributed under the terms of the Creative Commons Attribution License, which permits unrestricted use, distribution, and reproduction in any medium, provided the original author and source are credited.

Data Availability Statement: All relevant data are within the paper and its Supporting Information files.

Competing Interests: The Author(s) declare(s) no conflict of interest.

\section{Webinar \\ Stand on the Same Side Against Covid - 19: Ics-Containing Products in Treatment Covid- 19 Patients with Asthma and COPD}

This document is the direct transcription of a Webinar organized by Prof. L. Corbetta of the University of Florence on December $17^{\text {th }}, 2020$.

Scientific coordination:

Lorenzo Corbetta

Associate Professor of Respiratory Diseases - University of Florence

Scientific and Website Director of the European Association for Bronchology and Interventional Pulmonology (EABIP)

Organizing Secretary:

Consorzio Futuro in Ricerca

Via Saragat 1 - Corpo B - $1^{\circ}$ Piano | 44122 - Ferrara

cfr@unife.it

Translation Coordination and Editing:

Giorgia Biagini, MD

info@covid19expertpanel.network

Webinar's partecipants:

Prof. Lorenzo Corbetta - University of Florence

Prof. Leonardo M. Fabbri - University of Modena and Reggio Emilia

Prof. David Halpin - University of Exeter (UK)

Prof. Alvaro A. Cruz - Federal University of Bahia (Brazil)

Prof. Stefania Zanconato - Padova University Hospital

Corresponding author: lorenzo.corbetta@unifi.it 
“STAND ON THE SAME SIDE” Videoconferences

https://www.covid19expertpanel.network

"Implementing a science-based lockdown exit strategy is essential to sustain containment of COVID-19. China's experience will be watched closely, as other countries start considering - and, in some cases, implementing - their own exit strategies"

The Lancet, Volume 395, Issue 10232, 18-24 April 2020, Pages 1305-1314

This phrase expresses the purpose of this program called "Stand on the Same Side against Covid-19" that takes advantage of the new and rapid digital technologies to put together several experts worldwide. It's a global space were many countries hit by SARS-COV-2 can share only scientific information in order to face the pandemic.

\section{December, 17th 2020, INTERNATIONAL VIDEOCONFERENCE}

\section{"STAND ON THE SAME SIDE AGAINST COVID - 19: ICS-CONTAINING PRODUCTS IN TREATMENT COVID-19 PATIENTS WITH ASTHMA AND COPD”}

Lorenzo Corbetta: Good afternoon everybody, welcome to the sixth webinar of the project, 'Stand on the same side against COVID-19'. Today is ICS containing products in treatment for COVID-19 patients with COPD and asthma. I'm pleased to welcome again Leonardo Fabbri, the chairman and the co-chairman and Professor Cruz from Brazil, and the other new speakers participating to the project, Professor David Halpin, and Professor Stefania Zanconato. I give now the word to Professor Fabbri to introduce the next speakers, and I welcome again everybody.

Leonardo Fabbri: Yes. Thank you very much, Lorenzo, for your invaluable energy in organising these meetings, very pleased to join.

Thank you very much David for the very nice presentation. I think we have a couple of minutes for discussion, if there are any questions from the panel or from the participants, you can also send messages. You know today with the talking about COPD, there is an interesting controversy on the role of smoking, the protective effect of smoking that brings some crazy people to say, 'Treat COVID with smoking, or with nicotine as an anti-inflammatory agent.'

David Halpin: Yes, so I think I would support the WHO's view that actually the evidence is inconclusive at the moment. So, there's no conclusive evidence that smoking is protective, and equally there's no evidence that it's harmful. There are again some potential effects on ACE2 expression, but I think if we've got a neutral view on whether it protects or worsens COVID, we know smoking is bad for people. So, they should not start smoking just to try and protect against SARS-CoV2.

Leonardo Fabbri: Yes, there are people that I've seen are claiming that the burning of deaths due to smoking overcounts largely the risk of any protective effect.

David Halpin: The protective effect, yes, absolutely. Yes, I'm sure that that would be true.

Leonardo Fabbri: Thank you, any other questions?

Alvaro A. Cruz: David, very nice presentation, thank you. Do you have any information or evidence on those limits, or the benefits, of inhaled corticosteroids? Whether, let's say, in severe asthma where we need a much higher dose, would it still be beneficial, or could it be harmful?

David Halpin: I mean, I don't have any evidence. The data that I've shown you is all the evidence that I can find in terms of mechanistic studies looking at effects. I think we have clinical evidence about the differences, so, you know, the UK data that I showed you about risks of inhaled steroids, and showing in asthmatics that there was a dose response that those patients on higher dose seem to be at greater harm. But, you know, quite clearly that is confounded by the indication for the inhaled steroid in the first place. So, I don't think we have any data on whether there is a maximum dose benefit for inhaled steroids. My overall view is that I think that inhaled steroids are, probably, of neutral benefit. So, there are reasons why they may potentially be putting at risk, there are potential mechanisms that may help you protect, but overall it's neutral. So, I don't think there's any role in pushing the dose higher and higher to get that beneficial effect, because it may potentially have a greater effect on suppressing the innate immunity, and make you more at risk.

Leonardo Fabbri: Final questions, if there are others?

Lorenzo Corbetta: May I? One question we have an alert in Italy. There is a reported reduction in the use of treatments for asthma, COPD especially ICS. Have you noticed it in England, and what is the reason?

David Halpin: No, I think it hasn't happened, so the evidence that I have, and there's some quite nice data from a group called Propeller Health, who you all know is involved in remote monitoring, so they're involved in developing smart inhalers that monitor usage and feedback to patients. They also collect anonym data, and there was quite a marked upswing in compliance with medication early in the epidemic, so people, and in my experience my patients, they're frightened of developing the disease, they want to be as healthy as possible. So, they've been taking their 
medication much more regularly and, you know, according to what's recommended rather than forgetting to take it. I think there was an issue, as I mentioned that actually there was shortage of some inhalers because of the use outside respiratory disease so I think some patients did run into problems with access to medication but in my experience, if anything they've been taking more of it more regularly, rather than reducing it.

Leonardo Fabbri: Yes, while there is no evidence that asthmatics patients with COPD are at an increased risk of contracting COVID-19. How solid is the evidence that the presence of severe asthma and COPD is actually worsening the problem or is it increasing the mortality of the patient affected by COPD.

David Halpin: So, I know the data for COPD better than for asthma. There is now, I think evidence that if you have COPD in Europe or in North America, you're probably lightly more likely to end up in hospital with COVID but only a little bit more, so say an odds ratio of something like 1.2 whereas we know that age and other conditions have a much bigger impact so the odds ratio being over 75 is about five fold greater, so a much bigger effect than just having COPD. The best data that I know on outcomes is, again, UK data where we've got good linkage with primary care coding of co-morbidities and good ICU mortality data showing that in multi-variant models, neither asthma nor COPD are associated with increased ICU admission, nor increased mortality, so they're often with other co-morbidities or age that may put you at greater risk but in themselves, they don't appear to put you at greater risk and that's really a bit of a paradox, because we'd all think that people with impaired respiratory, particularly COPD where you've got a lot of permanent damage, you would do much worse if you ended up in ICU but that doesn't seem to be the case which is bizarre and interesting.

Leonardo Fabbri: Thank you.

Lorenzo Corbetta: Okay, I'm now pleased to introduce you Alvaro Cruz, from Brazil. Another expert on guidelines, he works a lot with GINA and GOLD and also with the Management of Asthma COPD during the outbreak in Brazil.

Alvaro A. Cruz: Hello my colleagues, I'm very pleased to be here with you all, however virtually, to share observations on the management of COPD during the outbreak in Brazil. I thank Dr Corbetta for the invitation to be part of this symposium, chaired by professor Fabbri, one of the major global leaders in the field of respiratory health of our times. And the panel of highly distinguished speaker, let me share my screen now. Well, these pictures, taken from the same position illustrate the enormous inequalities we face in Brazil. The dark side, challenging healthcare professionals, researchers and policy-makers. At the beginning of the

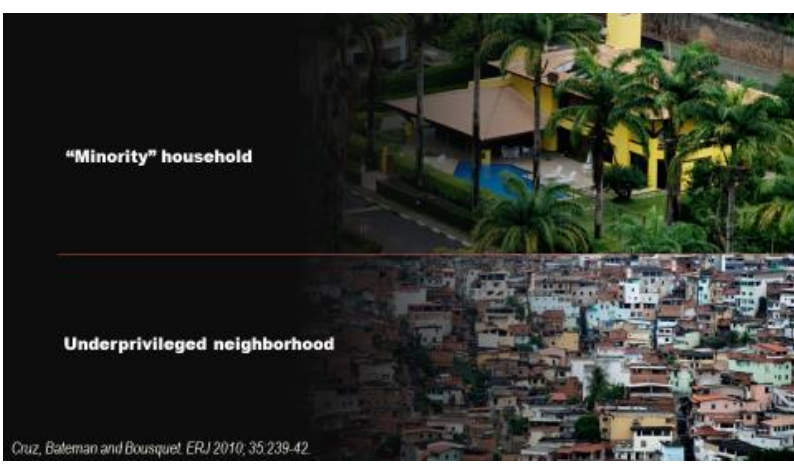

STAND ON THE SAME SIDE AGAINST COVID 19

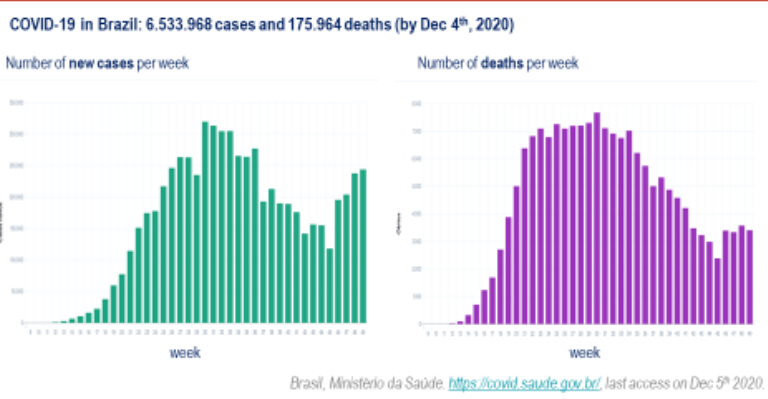

COVID pandemic, I was very worried of its consequences towards the underprivileged but I'm glad to report to you, we have free universal coverage by our national public health system which has responded well to COVID in general in spite of the absurd negationists' statements and behaviours of some political leaders and their followers. Regarding my conflict of interest, I must tell you, although I'm a member of the governing bodies of many organisations including the board of directors of GINA, I am not officially representing any of them. My presentation reflects solely my observations which I will present and my opinion. Well, I am going to try and cover these our topics that I mention are the problems. Asthma, COPD and COVID in Brazil. COVID-19 mortality, Asthma and COPD. Asthma in COPD exacerbation in times of COVID-19 and finally, why asthma and COPD exacerbations are reduced. While these are the official statistics of the Ministry of Health on December 16th, over $7,000,000$ cases and 180,000 deaths, a second wave is clearly starting, as you see, both in terms of number of cases and the deaths.

Mostly on the number of cases and in my position of front line physician, I see this as even higher, this is my perception. Still not appearing in the statistics, mostly of death. This is the picture of the prevalence of asthma in Brazil, of high prevalence. What we see here is a report of data generated by WHO survey of 5,000 adults which demonstrates the prevalence of asthma in adults in Brazil is $12 \%$. The same as what we have reported in the past. This is the prevalence of asthma, diagnosed. The figures for symptoms of asthma in the last year are around $23 \%$, both for adults and adolescents, highlighting the likelihood of a lot of under-diagnoses. This is the prevalence of COPD in five Latin American cities from 
Mortality due to COVID-19 in the State of Sao Paulo - Brazil $(n=42,788)$

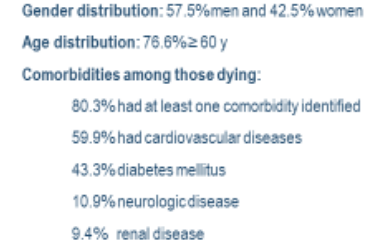

\begin{tabular}{l} 
Other comorbidities associated with deaths: \\
$8.3 \%$ any lung disease \\
$8.2 \%$ obesity \\
$5.5 \%$ immunossupresion \\
$3.1 \%$ asthma \\
$2.1 \%$ liver diseases \\
\hline
\end{tabular}

ASTHMA: number of severe asthma exacerbations ${ }^{1}$ in the ATTACK Study
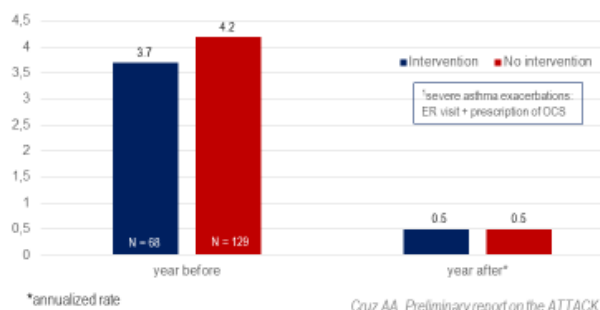

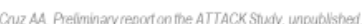

the PLATINO study which shows that in Sao Paolo, we have regions of the country. Now, doing the same for asthma, a nearly $15 \%$ adjusted prevalence, one of the highest in Latin hospitalisations in the public health system 2019 in dark blue America and fortunately, the proportion of smokers in Brazil then 2020 in red from April to September, six months period is not among the highest and in fact it's declining. Well now, of time. 45,000 hospitalisations last year. 19,000 let's look at the mortality of COVID in the state of Sao Paolo hospitalisations this year and the same trend in every one of looking at over 40,000 cases or deaths. The gender the give regions. Well, we should thank God that the much distribution is a little bit skewed towards males. The age feared storm did not hit hard patients with asthma and COPD. distribution, as expected of the deaths almost $80 \%$ above 60 years of age and the co-morbidities, $80 \%$ at least one comorbidity, $60 \%$ had quite cardiovascular disease. $43 \%$ diabetes. And we have here the lung disease, $8.3 \%$ have any lung disease, $3.1 \%$ have asthma. Its difficult to understand why they have split here COPD is in this group of any lung disease.

As I have just shown to you these prevalences are much lower than the prevalence of COPD and asthma in the population so we need to understand what is happening here. Now, I looked at the Ministry of Health's databases around COPD of hospitalisation in the Brazilian public health system comparing 2019 and 2020 from April to September and what we see here at your right, the total numbers 60,000 hospitalisations in 2019. 28,000 in 2020. It's less than half and this trend is exactly the same in every one of the five

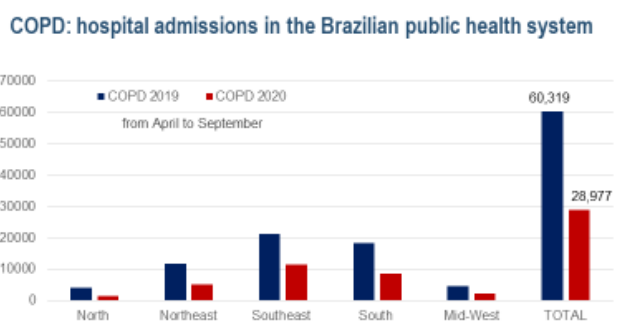

Brazil, Ministério da Saúde. hitto//tabnet, datasus gov brilast acceess on Dec fas 2020

STAND ON THE SAME SIDE AGAINST COVID 19

ASTHMA: hospital admissions in the Brazilian public health system

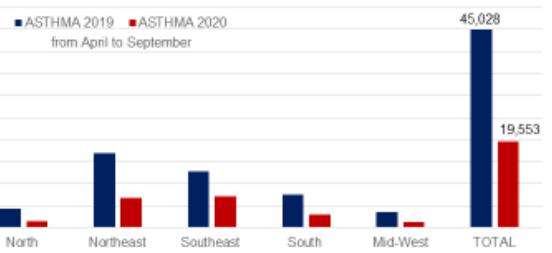

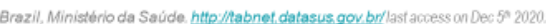

On the contrary, it seems to have spare them. What I presented is just database, retrospective information, now I wish to make some comments on some prospective observations. This is a report of data from our asthma cohort from ProAR and a comparison between this cohort and the UBIOPRED European cohort of adults which shows remarkable similarities in spite of so many differences we have. We have a special group with mild or with moderate to severe asthma followed up for several years, then despite of the disruption of the entire health system and our clinics, we haven't heard of many deaths so we followed some 1,000 people with moderate to severe asthma and we just heard of three deaths so far. This is another observation from our group, it's a pragmatic cluster randomised parallel group trial of the effectiveness of collaborative care for asthma in primary healthcare funded by NIHR. In this study, while we were looking at an intervention to build capacity of primary healthcare workers while looking at usual care. Well, we couldn't perform the study because there was no way we could build capacity in this chaos, we are living it so we just monitor these people with a systematic questionnaires by telemonitoring every three months and we were expecting to recruit 600 patients. We recruited only 200 . Now what I present here are the results of the follow-up of these 200 subjects, many of them have not completed one year of follow-up so we have here the number of severe asthma exacerbations in this study, we call ATTACK Study. The year before the initial group of this study was an episode of severe asthmatic exacerbation so this is what they had before, so an average of four sever asthmatic exacerbations defined by emergency room visit plus a prescription of oral corticosteroid.

In dark blue, the group in which we do the intervention but we didn't complete it and the red, the group in which there was no intervention, they would befall in usual care and what we see is that in the year after, using annualised rates, the average number of severe exacerbation went from four to 0.5 , a major reduction in exacerbation. 
STAND ON THE SAME SIDE AGAINST COVID 19

Possible explanations for the reduction in ASTHMA exacerbations in 2020

- Related to asthma, or its treatment: atopy reduces the risk of COVID

ICS prevents SARS-COV-2 infection ICS attenuates COVID-19

- Related to patient's behaviour: better adherence to treatment

Related to health system:

fear of attending ER due to COVID-19

reduced priority to asthm

reduced avalability of beds

STAND ON THE SAME SIDE AGAINST COVID 19

Possible explanations for the reduction in COPD exacerbations in 2020

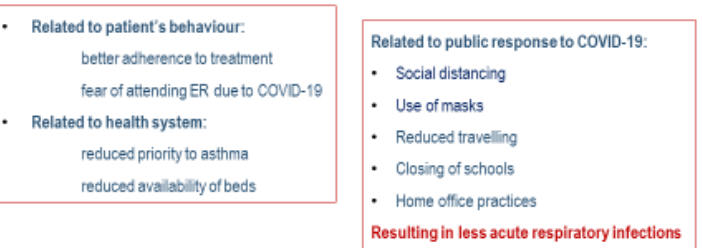

Well, let's see what can be behind this. Well, I put a lot of questions marks in everything I propose here which is speculation or hypothesis so these are the possible explanations for the reduction in asthmatic exacerbations in Brazil in 2020. Also these observations I just comment to you on our cohorts. Some are related to asthma or its treatment as well illustrated by David. Atopy reduces the risk of COVID? Some have proposed this. I see as prevents of SARS-CoV-2 infection. I see it attenuate COVID-19, is it possible? Related to patients' behaviour. Was there better adherence to treatment? There is some ecological data from pharmacies in Brazil to indicate that people use more corticosteroids or at least they bought more. Fear of attending emergency rooms due to COVID-19? We have reduced the rates of what we call 'severe exacerbations' in our studies or even reduced the number of people hospitalised due to asthma. And then related to the health system. Reduced priority to asthma? Reduced availability of beds? But also some related to public response to COVID-19. Social distancing? Use of masks? Reduced travelling? Closing of schools? Home office practices? These are all conditions or behaviours that might have resulted in mass acute respiratory infections which we know well are the major cause of exacerbations in asthma and COPD. Well, looking at the COPD possibilities, it's about the same, related to patients' behaviour, better adherence to treatment, fear of attending emergency rooms due to COVID-19, all question marks.

Reduced priority to COPD in the health system, reduced availability of beds and all the others relating to behaviours that could have resulted in less acute respiratory infections. Well, in summary, asthma and COPD during the COVID epidemic in Brazil, COVID has affected over 7,000,000 Brazilians in a country with a $12 \%$ prevalence of asthma and $15 \%$ prevalence of COPD in those over 40 years. There was

\section{STAND ON THE SAME SIDE AGAINST COVID 19}

Summary - Asthma and COPD during the COVID epidemic in Brazil

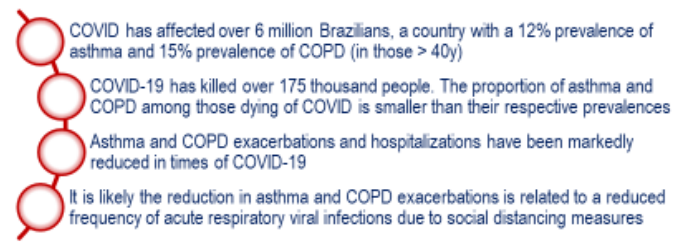

a thought that could have a potential of a lot of problems, hopefully it didn't happen. Although COVID as killed over 180,000 people, the proportion of asthma and COPD among those dying of COVID is smaller than their respective prevalences.

Asthma, COPD exacerbations and hospitalisations have been remarkably reduced in times of COVID-19. And finally, it is likely the reduction in asthma and COPD exacerbations is related to a reduced frequency of acute respiratory viral infections due to social distancing measures. I think it's time to seize the attention given to respiratory health globally to raise priority to asthma and COPD. No longer separate from infections, respiratory conditions but as a whole respiratory disease, or even better, respiratory health. We have compelling evidence from major reduction in exacerbations of chronic respiratory disease due to behaviour changes. I think this warrants major, immediate investment in research to figure out what can be applied to protect subjects with asthma and COPD after COVID? Thank you very much.

Lorenzo Corbetta: Thank you Alvaro for your very elegant presentation and if there are some questions from the panel? David?

David Halpin: Thank you Alvaro, that was really interesting. Have you got any data on COPD or asthma, probably COPD mortality during this time? Because in the UK, we've seen, again or saw back in the spring quite a marked reduction in hospitalisation but there was something like a $20 \%$ increase in mortality from COPD compared to previous years so it did look as though the explanation was people were frightened to come into hospital and were staying at home when they really should have come to hospital and one of the messages has been 'if you need to come to hospital, you should come to hospital, you shouldn't be afraid of COVID'. Have you got any similar data from Brazil?

Alvaro A. Cruz: David, the database for mortality in Brazil takes much longer to be built because there are many checks, so we don't have this available. For asthma, we thought of this but in our study, the one that I showed in primary healthcare, we've been calling these people and we have no evidence that they are heavily more taxed and stay at home because they have an action plan with oral corticosteroids and they are not using it, so it seems that they are really protected somehow. 
David Halpin: I mean I think all the things you talked about the masks, the working from home, the avoiding travelling all of those things are reducing viral infections aren't they? Which has to be a good thing but it did look in the UK certainly that the COPD patients were perhaps taking it just a step too far and not coming to hospital when they should have done.

Lorenzo Corbetta: Okay, if there are no more questions, now pleased to introduce Professor Leonardo M. Fabbri, everybody knows Leo Fabbri, an expert in asthma, COPD and with the presentation on the "Position of the guidelines".

Leonardo Fabbri: Thank you again for the invitation, title I've been assigned is very simple, the position of asthma and COPD guidelines, I have to admit that GINA and GOLD have no guidelines, have strategy documents and I highlighted the data.

Leonardo Fabbri: First I will discuss the GINA but before doing that I'd like to tell you and inform those of you who are not aware that on the 16th and 17th of November there was an international conference organized by GINA and GOLD in Philadelphia and the two days devoted one to COPD and one to GINA-, you may have access with the link below I leave this for you so that you can check that and you have the agenda that you can read now but if you click on the agenda once you are in you have registered that you are in and you can play all the presentations they're very informative and very nice but then in saying that, they're just an advertisement for GOLD and GINA that are very good and very active in the field of COPD and asthma. So, GINA already last March released a document and posted in several websites including the GINA website The Global Initiative for Asthma and making the following recommendation first to avoid useless, invasive, potentially dangerous measurements like spirometry and in general follow infection control procedures in far as so generating procedures where needed. These regard particularly carefulness required for nebulization, oxygen therapy including nasal prongs, sputum induction, manual ventilation, non-invasive ventilation, and intubation, and you can also have a link for this-, all the other ideal strategies are the same for COVID in general. The

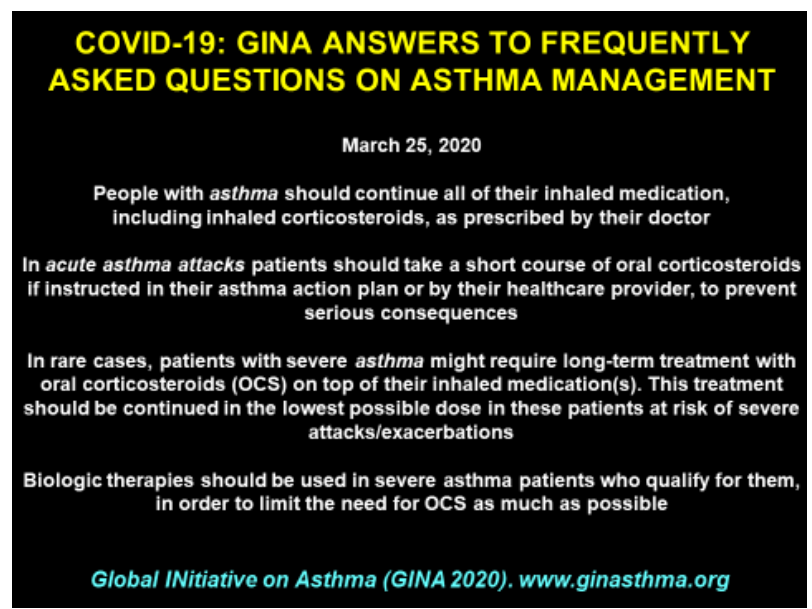

document was then updated with the recommendation that people with asthma should continue all their inhale medication including inhale corticosteroid as prescribed by the doctor, in acute asthma exactly the same including the inhaled bronchodilator with cautions for the potential dangers of using inhalation therapy and oral steroids is recommended and is required.

Sometimes people with severe asthma may require a longterm minimum dose of corticosteroids on top of their inhaled medication and all treatment should be continued. The patient should not interrupt the regular treatment when they take the oral steroids, and even if the evidence is very limited the recommendation is that even biologic therapies should be continued in this patient-, nebulizer avoided if possible or all confined, PMDI via spacer while the patient is treated for a severe attack their maintenance in asthma should be continued at home or in the hospital. Patients with allergic rhinitis should continue their nasal corticosteroids and again spirometry should be used only if strictly necessary, which in regular management of COPD and asthma is not really required. As David Halpin showed you convincingly, ICS should be written and corticosteroids should be used only in conjunction with exacerbations that is abuse of oral corticosteroids. We know that particularly associated with excessive use of PRN short-acting bronchodilator, the patient should be instructed, not only of the use of the medication but also in the plan and again avoid nebulization. The document also makes a recommendation for the so-called by GINA people, 'ACO asthma COPD', the GOLD people call it 'Asthma and COPD' not ACO, but basically the message is in this table continue to treat the patient as recommended for a patient who has not had COVID-19, so if asthma, it is asthma, if it is overlapped with the asthma, if it is only COPD without asthma take the COPD. Let me remind you, as I highlighted in the beginning because there are controversies in the original national guidelines, that the GINA is an evidence-based document that is prepared by GOLD by expert true evidence, true evidence taken from the literature. Some of the recommendations are off-label because in some countries the drugs are not registered so there is no use of 'off-label' in the GINA.

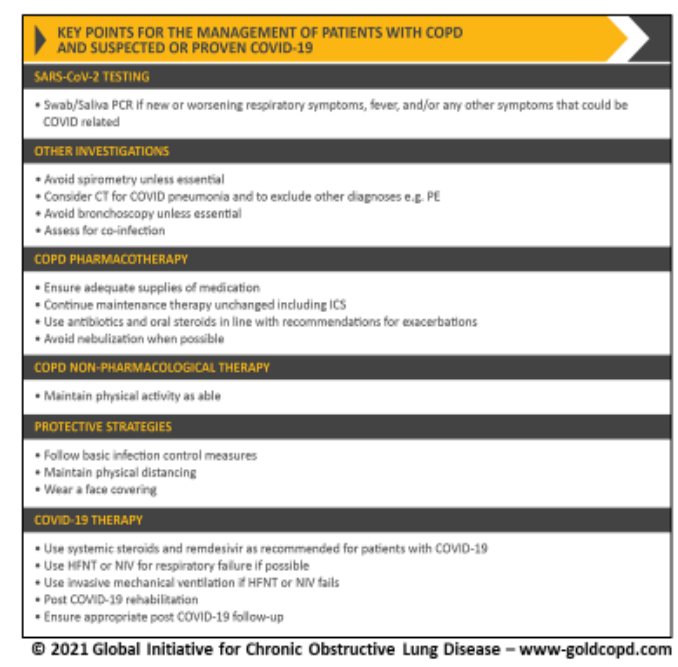


For new therapies, the recommendation are usually based on at least two or one solid randomized clinical trials providing evidence and for existing medication with evidence and new regiments the same applies for the rules and when you-, the most important part of this recommendation is when assessing and treating patients you should refer to your own professional judgement and take into account local and national guidance. GOLD, now above the document and the teaching slide labelled 20 and 21 are available in the GOLD website and the release of the document was anticipated by a very nice article published in the American Journal of Critical Care Medicine by David Halpin and other members of the scientific committee of GOLD.

The main question of the management of COPD is whether COPD are at increased risk of becoming infected with SARS, the answer is no, although the data is limited, ICS long-acting bronchodilators, roflumilast, macrolides should be continued when required likewise systemic steroids with antibiotics during exacerbation. A particular, careful, clinical approach should be taken to make the differential diagnosis between an exacerbation of COPD and COVID, usually they are different but they may also present with very similar characteristics. Patients who develop moderate to severe COVID-19, including hospitalisation should be treated with evolving pharmacotherapy and-, now the slide is still updated on the document but there are some controversies on the cost benefits of remdesivir, those are matters for the severe patient and anticoagulation also when you have a suspicion of coagulation unless the patient is lying in bed for a long time. The acute respiratory failure should include appropriate oxygen supplementation and noninvasive ventilation, you have to look at the specific recommendation but again, protecting lung ventilation in patients with ARDS.

In a patient that's developed mild COPD, they should be just treated with the symptomatic drug as normal, whereas the patients who develop moderate and worse COPD should be monitored more frequently than normal with particular attention to the need of oxygen therapy. You know, there is a common experience that you have a patient coming in with

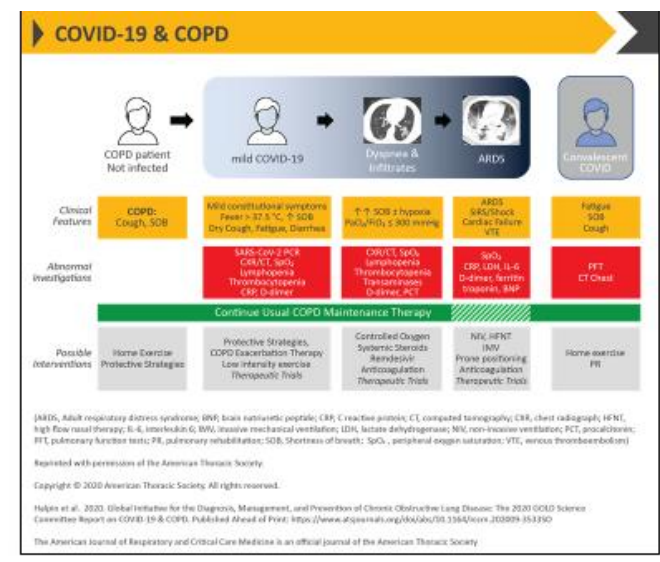

(๑) 2021 Global Initiative for Chronic Obstructive Lung Disease - www-goldcopd.com some dyspnoea symptoms more relevant than the saturation but you should be watching very carefully the patient admitted to the hospital because they may develop respiratory failure within hours. These are the slides that are included in the 20 and 21 GOLD document, protective strategies are the same for everybody including considering, shielding and sheltering in place of the patient, no spirometry, make sure not only that the drugs are properly taken but that they are also made available according to the treatment plan and no pharmacologic therapy if possible should be continued, including the influenza vaccination-, and this year there is a recommendation from the document also of Pneumococco vaccination. If the patient's contract or are suspected of contracting COPD you should go through the usual PCR diagnostic approach, swab oral saliva and use recommendation and pharmacotherapy and nonpharmacotherapy protective therapy and the COVID-19 therapy. This is the most instructive and carefully designed, simple approach to the different COVID-19 affecting COPD patients. On the left you have the COPD non-infected that you follow the usual ways if it is milder you have most importantly, you have to be watching the signs or symptoms that predict the development of respiratory failure, here our report has listed.

And if by contrast, the patient developed more dyspnoea particularly and hypoxaemia then you have to increase the diagnostic approach and if there is respiratory failure you should treat the patients with the usual approach for respiratory failure with all the valuables that are included. The convalescent COVID patient should be carefully followed up and the GOLD document includes also a COPD follow-up checklist, that should be monitored. I think that any unit is now following a strict protocol to monitor these patients that may develop, there is no evidence yet, it may be a matter of discussion, of developing of fibrosis as immediately as we said but if we look at the experience with the ARDS it's not so common the developing of non-reversible hydrolysis after an acute event. This is the end of my presentation, I am happy to answer any questions.

Lorenzo Corbetta: Thank you, thank you Leo for a very nice presentation. Are there some questions from the circle team? I have one, in the last figures that you showed I can't see any difference between COPD patients and healthy patients, are there some differences? And suggestions that you can give us?

Leonardo Fabbri: Well usually, it may present with cough and dyspnoea but most of the cases of COVID-19 present with dyspnoea and fever and also as you know, taste and smell abnormality, gastroenteric systems, it's more systemic. I mean the COPD exacerbation is an increase of dyspnoea, $100 \%$, the cough is producing sputum, $20-30 \%$, rarely a fever and so I think that in the prototype they actually are not so difficult to distinguish but equally during the pandemic like now, I think that my colleagues in the clinic, they almost avoid imaging because they say that the characteristic feature of COVID-19 are almost invaluably present symptoms that I mentioned to you and in fact, they don't even do chest $\mathrm{x}$-ray or most 
importantly CT-scan because they now have the ultrasound next speaker. I'm now pleased to introduce Stefania that helps them to avoid going to the imaging department, Zanconato who is the head of pneumology and paediatric particularly for CT-scan I have to say, chest x-rays are much easier to be done.

David Halpin: It is important, I think you're absolutely right Leo and what we've said in GOLD certainly is, to answer Lorenzo's point is that actually, the management of a COPD patient should be no different to the management of any other patient but I think one of the things that have become clear with COVID is the high risk of venous thromboembolism, so I think if a patient is quite significantly hypoxemic then a CT looking for pulmonary embolism is an important investigation for those patients. So, having a high suspicion for thromboembolic disease applies to all patients but rather than just managing the lungs we need to think-, as your very aware-, about the pulmonary circulation as well.

Leonardo Fabbri: Yes, you're right, almost all the patients at least in my country that are admitted to the hospital and are predicted to stay for more than two or three days in bed are treated with low molecular weight heparin prophylaxis that doesn't mean that you don't have to make the, you know, proper diagnosis of potential pulmonary embolism because we know that in COPD is increased but not that much, I mean it's not a common feature whereas in COVID-19 not only may be frequent but may even be more complicated than that because one question I didn't have the time to ask you, David, during your presentation is that the two tissues that are actually targets of the COVID-19 are the epithelium first but the endothelium second and if you look, there is a couple of papers, two or three papers of pathology that shows that it's not only embolism it's also vasculitis that makes the picture more severe, you made a very good point.

Alvaro A. Cruz: Leo, I think it's pretty clear that COVID spares the airways, it enters through the airways but it hits the lungs and the vessels but spares the airways. Do you have any explanation about this or any further information on how things work in terms of the information in the airways?

Leonardo Fabbri: Yes, another very interesting question. There is a case report of COVID-19 deaths or of an asthmatic who died of COVID-19 and he had all the characteristic features in the airways of asthma whereas he had all the characteristic features of COVID in the lungs. So, the second point is that-, I'll send you the reference when this meeting is over. There is an interesting review article on COVID-19 as a systemic disease and just highlighting that the endothelium is probably the most relevant and that simultaneous abnormalities are present particularly in the liver, in the kidney, in the brain and so that why the airways are spared I think that goes together to the fact that people with asthma have not increased the risk of getting COVID-19 probably, you know, either for the defence mechanism that David highlighted or the protective effect of treatment might be preserved, protected maybe, but there is no evidence.

Lorenzo Corbetta: Okay, thank you. We can move to the allergology unit, the Department of Women's and Child Health of the University of Padova. Thank you Stefania for accepting to participate.

Stefania Zanconato: Okay, hello. Thank you for the invitation to this webinar I will talk from the paediatricians side. So, I'll start just with what we know about COVID-19 and children. This recent data from the WHO is on the distribution of confirmed cases by age. As you can see, only two percent of all cases were reported in children under four years of age, and five percent in children under fourteen years of age. So, COVID-19 is reported much less frequently in children than in adults. This data from different countries, this is the initial data from China, in 2135 children with COVID19 and as we can see most of the cases, most of these children are mild or asymptomatic disease, mild or moderate disease. (https://doi.org/10.1542/peds.2020-0702)

So, symptoms were less severe than in adults, and young children, especially infants, were more vulnerable to severe SARS CoV-2 infection. This data from different European countries, you see from 82 health care institutions, in 582 children with a median age of five years and as we can see 48 of these children required the ICU admissions and fifteen percent of these children that went to an ICU were under one month of age, and $52 \%$ had previous medical conditions. So, the risk factors for ICU admission in the paediatric age are very young age, under one month of age, male sex, preexisting medical conditions and lower respiratory tract infection signs at presentation. This data from different countries, the data more or less reports similar results. (https://doi.org/10.1016/S2352-4642(20)30177-2)

Children were one, two percent of all the diagnosed cases, $4-24 \%$ were asymptomatic and death rates were up to $0.7 \%$. In our paediatric hospital since February we admitted 61 children, at least until the day before yesterday. 61 children with SARS CoV-2 infections. 44 were under one year of age, $38 \%$ had previous medical conditions and none had chronic respiratory diseases and none needed ICU admission. So, children are more often asymptomatic, have less comorbidities, so why do children have milder presentation? There are different hypotheses.

One is that ACE2 receptors are less mature in the airways of children. That in children there are different, you know, children usually have many viral diseases and so SARS CoV-

\section{MILDER PRESENTATION IN CHILDREN}

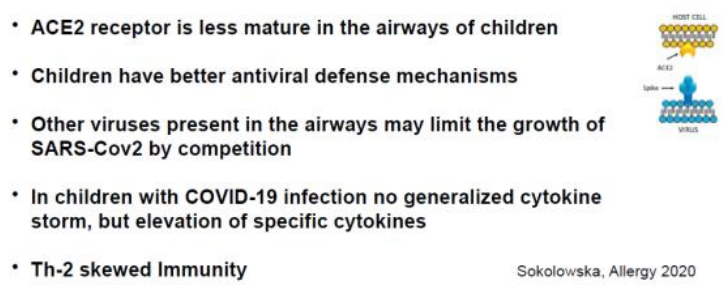


STAND ON THE SAME SIDE AMAINST ROIVID 19

COVID-19 in the Pediatric Population-Review and Current Evidence

Indirect effects of coronavirus epidemic in children

Higher rate of psychiatric morbidities

Education loss

Unhealthy lifestyle changes

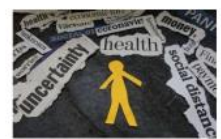

Increased child neglect

Rabinowicz, Curr Infect Dis Rep 2020:22:29

$11 \%$ of the paediatric population has asthma, and we see that out of 2000 people that were diagnosed with COVID-19, only 40 were children and out of these 40 children only two were allergic and one had asthma. So, children with allergy and asthma are rare in the COVID-19 population and this suggests that allergy could be a protective factor. This data from 182 hospitalised children from China, 42 had allergic diseases, $98 \%$ had no severe disease and what we can see there was no difference in allergic prevalence within children with pneumonia and with no pneumonia and there was no difference between allergic and non-allergic COVID-19 children in disease incidence, clinical features, laboratory and

2 has to compete with other viruses in the airways of children. Then, probably in children there is no generalised cytokine storm and the children have more often a T2 skewed immunity that seems to be protective.

So, what do we know? We know that COVID-19 is reported much less frequently in children than in adults, the children have a much milder disease than adults but unfortunately there is increasing evidence of a multisystem inflammatory syndrome post COVID-19, about a month after the COVID infection and SARS CoV-2 infections. It is a Kawasaki-like disease with persistent fever, elevated inflammatory markers, multi-organ involvement, cardiogenic shock. Right now we have two of these children admitted in our hospital, one of them required ICU admission. But we cannot forget as pediatricians the other effects of the Coronavirus epidemic in children. The higher rate of psychiatric morbidities, education loss, the unhealthy lifestyle changes, we know they don't exercise, they spend a lot of time in front of a cellular phone and they eat junk food and finally, the increased child neglect.

But what do we know about asthma and COVID-19 in children? At the beginning of the pandemic, you know, the CDC stated that, 'Asthma is a prognostic factor for COVID19 outcome such as morbidity and mortality' but right now we know that initial concerns about children and young people with asthma being particularly affected by COVID19 has not been realised.

On the opposite, it looks like children and adolescents with allergy and/or asthma seem to be protected from SARS CoV2 infection or from COVID disease. But this is a study that evaluated the expression of the SARS CoV-2 receptor, the ACE2 receptor, the upper airways of 318 children were part of a quarter of children and they had the nasal brush and we can see here on the left the ACE2 expression was decreasing the nasal epithelium of children with allergic sensitisation. Why? In asthmatic children there was a progressive lower level of ACE2 according to the degree of IgE sensitisation among children with asthma. So, paradoxically, asthma may be protective, as the ACE2 receptor may be underrepresented in the lungs of atopic children. (https://doi.org/10.1016/j.jaci.2020.04.009)

This data from a couple of areas from North Italy is where we can see $30 \%$ of the paediatric population is allergic and

immunological findings.

(https://doi.org/10.1016/j.arbres.2020.07.003)

This data from Spain where 29 asthmatic children with COVID-19 were compared, or maybe with SARS CoV-2 infection were compared with 183 asthmatic children with no infections and all these children had mild symptoms of SARS CoV-2 infections, and there were no difference in lung function and asthma control between the asthmatic children with SARS CoV-2 infections and those who did not have infection. So, in asthmatic children COVID-19 symptoms were mild, and allergic asthmatic children are not more vulnerable to suffer from COVID-19. This data from London, when there was the lockdown and the schools closed we see the dramatic decrease in emergency department attendance of children with asthma or wheeze and even though the pollution measured by PM10 levels did not change. So, in closing schools, children may have reduced exposure to viral infections, we have already talked about this and pollution on the school run and have undertaken less formal exercise.

These are some of our data where we evaluated 92 asthmatic children with the same prescription for asthma in the same month of Spring 2019 and Spring 2020 during the lockdown, and we see that there was a better asthma control with a lower GINA score in the year 2020 compared to the year 2019 and we had less acute exacerbations, so better control of asthma and finally, this data from Philadelphia that I think would be applied from many different countries. What happened during the pandemic was a dramatic decrease in the in-person, outpatient care for asthma with an increase in telephone and video telemedicine. In the same time there was an important decrease in rhinovirus infection that we know is one of the main triggers of asthma exacerbation in children and a decrease in (mw 01.20.59) in the systemic steroid prescription.

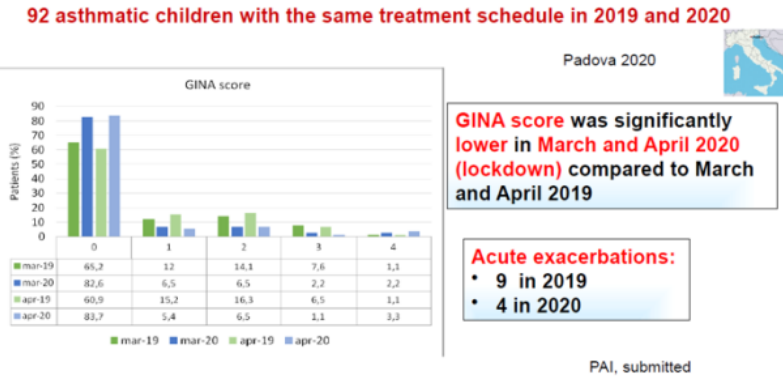


So, the care of children with asthma changed I think all over the world during this pandemic and with more video telemedicine, less asthma in-person care, less systemic steroids, less rhinovirus and less exacerbation. Finally this is the last data. This data from 99 paediatric centres all over the world, and they report similar results. The virtual clinics were launched in most centres, better than expected control was reported in $20 \%$. Adherence, as we already heard in the previous talks, appeared to increase and there were only fifteen confirmed cases of COVID-19 that were reported, so similar to that of general paediatric cohort. So, children with asthma were not disproportionately affected by COVID-19 and clinical services have rapidly responded to the pandemic. Obviously only ongoing epidemiological studies will tell us the long-term risk of COVID-19 on children with asthma. These are the commendations released by the EAACI on asthma and COVID-19, or allergy and COVID-19. They say that paediatric allergists are dealing with a patient population that is at low risk for COVID-19. That like in adults, there is no scientific evidence that allergy and asthma treatment increases susceptibility or severity of COVID-19 disease, and asthma control with appropriate medications should be a major goal in all asthmatic patients, children and adults. What about children with severe asthma? As we know, there are not so many studies focusing on severe allergic phenotypes and particularly obviously in children. What we know is that in case of an active SARS CoV-2 infection, biological treatment needs to be stopped until recovery. What about asthma treatment-, which treatment they have to follow, children and adolescents? You all know that since 2019 that GINA no longer recommends SABA-only for treatment for step one.

So, adolescents and adults, step one the first choice recommended, the first therapy, the first choice is the low dose ICS-formoterol that can also be used as step two as an alternative to daily low dose of inhaled corticosteroids. In children, on the other end, step one, a low dose of ICS is recommended whenever short acting beta agonist is taken while step two, the first choice, daily low dose inhaled corticosteroids.

What else can the paediatrician do? The paediatrician can help his children particularly, his adolescents. Try to help these children comply with treatment. We know that adolescents are very bad patients and also we know that children don't use their drugs, their asthma drugs in the correct way. So, paediatricians need to monitor and correct

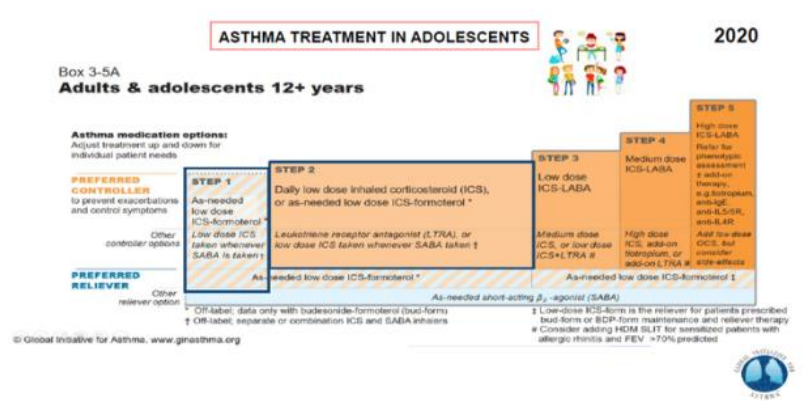

\section{STAND ON THE SAME SIDE AGAINST COVID 19}

Coughs and Sneezes: Their Role in Transmission of Respiratory Viral Infections, Including SARS-CoV-2

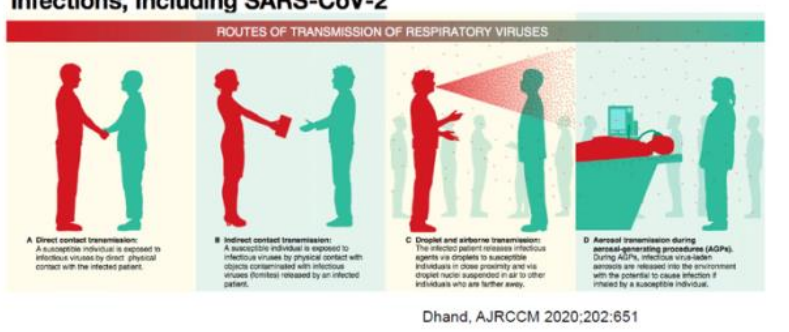

inhaler techniques at every opportunity. Just a couple of words on what happened when the children went back to school? These are just some emails parents sent to us,

"Dear Doctor, my child does not want to wear the face mask, she can't breathe."

"Dear Doctor, my child does not want to wear the face mask, she can't breathe........"

"Dear Doctor, I am very worried for my child who has asthma. Is he at risk for COVID-19?"

"Dear Doctor, Today my child has been sent home from school because she was coughing, what can I do?"

And we really were overwhelmed with these phone calls and emails. What about face masks in children? The WHO states that, 'Face masks of breathable material, worn properly, will not lead to health problems.' So, even in children you see, for most children masks won't make it harder to breathe. So, children over two years of age have to wear a face mask. This includes children with many medical conditions. What about nebulisers? We already heard what GINA tells us and we know that the main route of SARS CoV-2 transmission is direct contact, indirect contact.

The main respiratory transmission is through droplets, while the transmission of aerosol-producing generated procedure is not clearly stated. So, at present there are limited data on whether nebuliser administration may be considered an aerosol generating procedure or may represent a transmission risk and we know that many children, particularly with the acute exacerbation, use nebulised treatment.

We know we have also different advice. We already heard that GINA advises against the use of nebulised treatment where possible. CDC does not advise against the use of

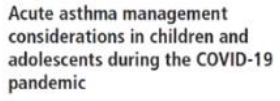

Although the risk of infection spread from a child undergoing nebulizer therapy to healthcare workers is not quantifiable,

$$
\text { P }
$$

the current available evidence supports that the risks are very low as the droplets are presumed to originate from the nebulizer device

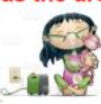
and not from the patient

Nagakumar, Arch Dis Child 2020 
UK this week about a new variant that's been identified in the South East of England and around London which seems to

\section{CONCLUSIONS...}

- COVID-19 appears to have less effect on children's health than in adults

- Asthmatic children seem to have low risk for COVID-19

- Asthma patients should continue taking their prescribed asthma medications

- If possible, switch from nebulization to MDI/VHC treatment

- Follow the prevention measures (face mask, hand hygiene, s distancing)

Do not forget the psycological impact on children and adolescents

nebulised treatment but states that there is an unknown-, we don't know what the exposure is for health care personnel that has still to use the PPE. NICE recommends to continue use of nebulised treatment and EAACI states that, 'In patients infected by SARS CoV-2, nebulisation increases the risk of virus deposition in the lower airways and should be replaced by spacers.' So, this is my last slide. What can paediatricians do? Paediatricians, what else can they do? Where possible-, obviously because if we find ourselves in front of a two-year old child screaming and crying, we do the best we can, but where possible we have to try to switch from nebulisation to MDI, obviously with a valved holding chamber in children. These are my conclusions.

Lorenzo Corbetta: Thank you very much Stefania. Very, very interesting presentation. Some questions from the panel?

Leonardo Fabbri: May I? First, thank you very much for the very clear lecture and nice to see you again after some time in Padova. The question is a naïve question. Every newspaper says that the children kill their grandfather, grandparents, because they bring infection home. Is that evidence-based?

Stefania Zanconato: Yes, let's say most of the children are infected in the family actually, not in their social environments. So, while we do not know how infection children are, while we know that children have the mild disease, that children are not often infected, we still don't know how contagious the children are. While most of the children, even now in our hospital, are children that came from family where there was an infection so they did not get infected at school but in the families.

Leonardo Fabbri: Thank you.

\section{Lorenzo Corbetta: Other questions? David? No?}

David Halpin: There is a question on the chat. So, we do talk about this in the goal document and I think that there is evidence both for reactivation and for reinfection. It's rare, but it happens and it seems to be generally in people that have immunosuppression either because of medication or because of immune deficiencies. So, I think it is something we should be aware of but it doesn't seem very common. In terms of new mutations, I mean, there's been a lot of publicity in the have a greater propensity to spread, it probably doesn't cause any worse disease but it seems to be transmitted more easily, and earlier in the year there seemed to be a different variant that emerged in Spain. So, this is what you would expect with a virus, that there is mutation and new variants arising. As I understand it, and has been stated in the UK, this won't influence the efficacy of the vaccine but it does change slightly the dynamics of transmission.

Lorenzo Corbetta: Yes, I completely agree. In our last webinar we had a biologist, Duccio Cavalieri, that confirmed that there is a new mutation that increased infections but not the pathogenic and the response to the vaccination.

Stefania Zanconato: That is important.

Lorenzo Corbetta: Yes. Okay, Leo, do you want to say something to conclude?

Leonardo Fabbri: Thank you. I think we had a very interesting webinar, we learned from each other. I hope Lorenzo you continue with your organization. The last was on vaccine, today we had very nice lectures on the ICS with the user, we heard about the children, we learned about the children and Alvaro as usual gave us a sparkling clear picture of Brazil, we are very concerned about Brazil but I think that in terms of percentages, we are now more similar, you know, and we hope that-, I think that the UK already started vaccination, they said in the press today that we should have the first before the end of the year on the 28th or 29th of December, and I don't know when in Brazil and I hope that these will rescue us from future disaster. Let me take the opportunity not only to thank you all for your enthusiastic participation but also wish you all and your families a merry Christmas and happy new year. Enjoy, bye bye.

Stefania Zanconato: Happy new year, yes. Bye bye.

Lorenzo Corbetta: Goodbye and thanks again. 